\title{
Capacity Optimization of the Intersection with Reverse-variable Lane
}

\author{
Meng Cao* \\ School of traffic and Transportation, Lanzhou Jiaotong University, Lanzhou 730070, Gansu, China \\ ${ }^{*}$ Corresponding author
}

\begin{abstract}
This article's aim was to study the effects, which were come from the length of reverse-variable lane, left-turn traffic arrive rate, left-turn green time and the reverse traffic arrive rate of the upstream intersection in the short-line grouped intersections, about the capacity of intersection,by Cellular automata model . The simulated results indicate that there was a critical value of traffic arrive rate. When the traffic arrive rate less than critical value, the reverse-variable lane could not improve the left-turn capacity. When traffic arrive rate greater than critical value, there was a optimal length of reverse-variable lane which can make the left-turn capacity been maximum. When the left-turn green time increased, the optimal length of reverse-variable lane been less. When the traffic arrive rate and the length of reverse-variable lane are certain, there is a minimum green time, which make the past-traffic flow reach the capacity. When the length between intersections is certain and the reverse traffic arrive rate of upstream intersection reach to a certain value, the function of reverse-variable lane and the left-turn capacity of intersection are been effected.
\end{abstract}

Keywords-traffic engineeringt; capacity; revese-variable lane; green time; traffic flow; lenrth between intersection

\section{INTRODUCTION}

Recent years, the quantity of vehicle is increasing year by year. And traffic jam is becoming a normality, which caused economic losses, energy waste, air pollution and traffic accidents. Government are always developing the public transportation, advocating the scientific construction of road network and attaching importance to the planning and design of urban road intersections[1], in order to resolve the traffic jam.. Researchers found that they can not improve the left-turn capacity of some intersections, by setting left-turn waiting areas, lengthening the green time or adjusting signal phase. Cause the restrictions of intersections' space and cannot increase the road to improve the left-turn capacity. The reverse-variable lane, which can make full use of intersection space and be used in some cities, to relieve the left-turn traffic pressure. At present, there is less research on reverse-variable lane. Shang Zhen-hua[2] putted forward setting method of the reverse-variable lane in the urban intersections, which include the conditions of setting reverse-variable lane, the limited length and research on supporting facilities of reverse-variable lane. But the length of reverse-variable lane, traffic arrive rate, left-turn green time and the reverse traffic arrive rate of the upstream intersection in the short-line grouped intersections, which not yet researched compresive--ly, can also influence the effect of reverse-variable lane.

Therefor, author decide to research the influence that the length of reverse-variable lane, traffic arrive rate, left-turn green time and the reverse traffic arrive rate of the upstream intersection in the short-line grouped intersections, make to reverse-variable lane, by using cellular automata model. Research found that there is a critical condition of setting reverse-variable lane and there is a minimum green time which can make the past-traffic flow be the maximum value. Paper put forward the method of setting the reverse-variable lane for maximum capacity.

\section{A. Definition of Reverse-variable Lane}

Reverse-variable Lane is set for improving left-turn capacity, by using adjacent exit lane as left-turn lane. The Figure.1 show the traffic channelization design about reverse-variable lane.

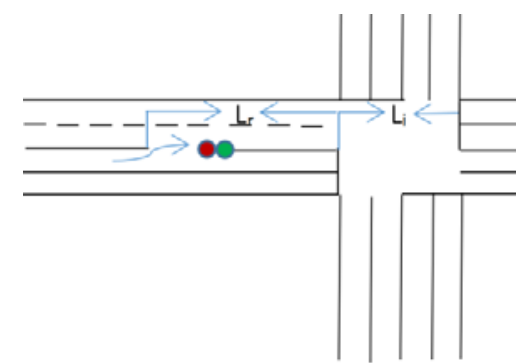

FIGURE

REVERSE-VARIABLE LANE

The opposite phase of reverse-variable lane is shown as Figure.2. In order to explain the paper, author use the intersection's traffic channelization design to research the problem about reverse-variable lane, which setted for left-turn vehicles.

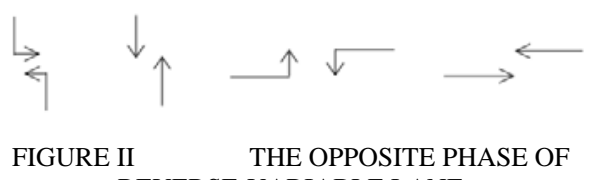

REVERSE-VARIABLE LANE

\section{B. The Description of Using the Reverse-variable Lane}

(1) The process that vehicle enter the reverse-variable lane: the green light on the reverse-variable lane's entrance, lighted later $t_{\mathrm{g}}(\mathrm{s})$ than the lighted time of the left-turn red light of the vehicles from north to south. $\mathrm{L}_{r}$ is the length of 
reverse-variable lane. And the $L_{i}$ is the east-west length of intersection. $\mathrm{V}(\mathrm{m} / \mathrm{s})$ is the average speed of the vehicle which driven from the stop-line to the entrance of reverse-variable lane. Therefor the $t_{g}$ can be calculated by the follow equation.

$$
\mathrm{tg}_{\mathrm{g}}=\left(\mathrm{L}_{\mathrm{r}}+\mathrm{L}_{\mathrm{i}}\right) \div \mathrm{V}
$$

(2)The waiting process of the vehicle in the reverse-variable lane: the waiting vehicles wait for the turn-left green light of intersection is lighted.The process that the vehicles leave the reverse-variable lane: because the vehicles occupy the exit lane of reverse traffic flow, the vehicles in the reverse-variable lane must be clean, when the left-turn traffic light of intersection is beginning to show red. Therefor the the red light on the reverse-variable lane's entrance, lighted before tr (s) than the lighted time of the left-turn red light of the left-turn vehicles from west to east. $\operatorname{Vr}(\mathrm{m} / \mathrm{s})$ is the average speed of the last vehicle in the reverse-variable lane. The tr can be calculated by the follow equation.

$$
\mathrm{t}_{\mathrm{x}}=\mathrm{L}_{\mathrm{r}} \div \mathrm{V}_{\mathrm{r}}
$$

This paper will apply the above control method into cellular automata model to research the problem of reverse-variable lane.

\section{Cellular Automata Model}

This paper chose the NS evolution rule to make research.

Step 1 Acceleration process

$$
\mathrm{V}_{\mathrm{i}}(\mathrm{t}+1 / 3)=\min \left[\mathrm{V}_{\mathrm{i}}(\mathrm{t})+1, \mathrm{~V}_{\max }\right]
$$

Step 2 Deceleration process

$$
\mathrm{V}_{\mathrm{i}}(\mathrm{t}+2 / 3)=\min \left[\mathrm{V}_{\mathrm{i}}(\mathrm{t}+1 / 3), \mathrm{d}_{\mathrm{i}}(\mathrm{t})\right]
$$

Step3 Randomization slowdown process with variable probability

$$
\mathrm{V}_{\mathrm{i}}(\mathrm{t}+1)=\max \left[\mathrm{V}_{\mathrm{i}}(\mathrm{t}+2 / 3)-1,0\right]
$$

Step 4 Vehicle Position update process

$$
\left.\mathrm{x}_{\mathrm{i}}(\mathrm{t}+1)=\mathrm{x}_{\mathrm{i}}(\mathrm{t})+\mathrm{V}_{\mathrm{i}}(\mathrm{t}+1)\right]
$$

$\mathrm{V}_{\mathrm{i}}(\mathrm{t})$ is vehicle's speed at $t$ time step. $\mathrm{x}_{\mathrm{i}}(\mathrm{t})$ is vehicle's position. $\mathrm{d}_{\mathrm{i}}(\mathrm{t})$ is distance with the vehicle number with $i+1$.

\section{NUMERICAL SIMULATION AND ANALYSIS}

Cellular automata model is a discrete model. The numerical is discrete,too. In order to analyse the simulation result clearly, this paper put the discrete numerical result into continuum figure.

\section{A. The Critical Condition of Setting Reverse-variable Lane}

Under the fixed arrive rate of left-turn vehicles, the simulation result of NS evolution rule about intersection's left-turn flow with a reverse-variable, and without reverse-variable lane presented as Figure.3.

From Figure.3, we can clearly observe that the two curves have a overlap, which mean that there is no mean to set a reverse-variable lane, when the arrive rate is lower than a certain value. From the lane without reverse, we note that the left-turn flow will not be more large with arrive rate's increase, when the arrive rate reach six hundred and thirty veh/h. The coming condition is left-turn traffic jam, if there are noting done to it, when the arrive rate is getting larger. So from the lane with reverse-variable lane, we know that the left-turn flow can be larger with the arrive rate's increase, even the value of it has exceeded six hundred and thirty veh/h. Therefor, from the contrast of the two curves, we can draw the conclusion that reverse-variable lane can improve the left-turn flow, and there is a critical value of arrive rate which decide if we should set reverse-variable lane or not.

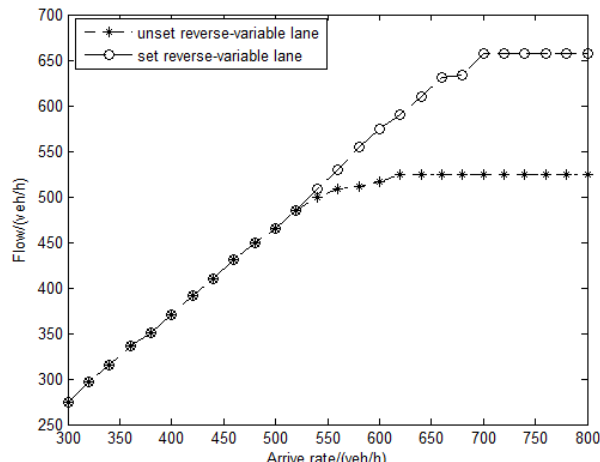

FIGURE III THE PASTED-TRAFFIC FLOW OF UNSET AND SET REVERSE-VARIABLE LANE

B. The Relationship between the Length of Reverse-variable Lane and Capacity

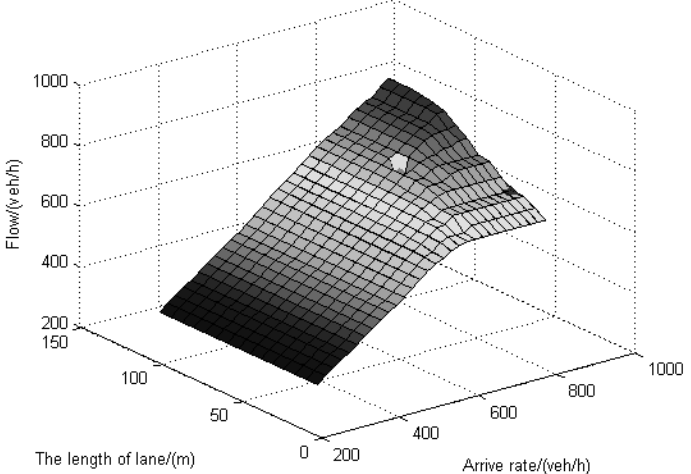

FIGURE IV RELATIONSHIP BETWEEN THE LENGTH OF REVERSE-VARIABLE LANE AND LEFT-TURN CAPACITY

From Figure.4, we can see that the left-turn flow will increase with the larger value of arrive rate of left-turn vehicle, when the length of reverse-variable lane keep a certain value. Because of the limitation of reverse-variable 
lane's length, the the left-turn flow will be a certain value(a) with the increasing of arrive rate. When the length of reverse-variable lane increase, $a$ will be larger . Because of the limitation of arrive rate, when the length of reverse-variable lane reach a critical value $(b)$, the left-turn flow will not increase any more. So, we can use $b$ as the most appropriate value, when we set a reverse-variable lane.

\section{The Relationship between Green Time and Capacity [3-5]}

From Figure.5, when the green time is certain, left-turn flo- -w will increase with the increasing of reverse-variable lane's length, until the length reach a critical value $(c)$. So, $c$ is the most appropriate value of reverse-variable lane, under this certain green time. When the green time increase, $c$ will be less with the reason that more vehicles can pass the intersection within a longer green phase time and the arrive rate is not change in this time.

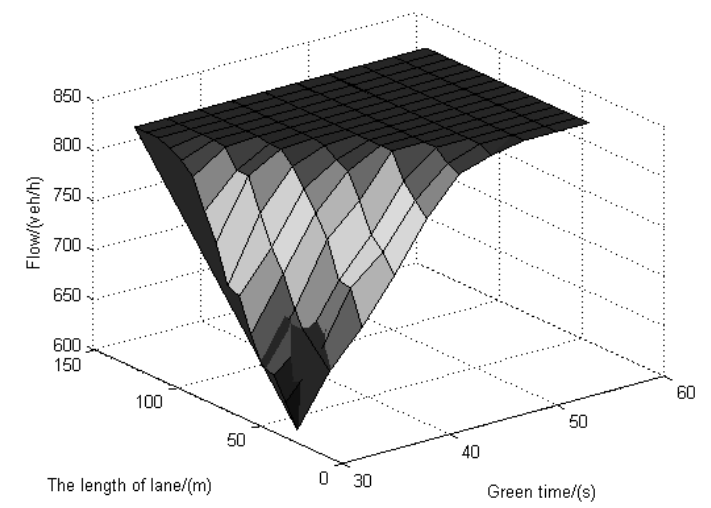

FIGURE V RELATIONSHIP BETWEEN LEFT-TURN GREEN TIME AND LEFT-TURN PASTED-TRAFFIC FLOW

D. The Relationship between the Arrive Rate of Upstream Intersection and Capacity

The road traffic is designed to study the traffic network at a global point of view. So it necessary to study the traffic problem at the upstream intersection, when studying the relationship between the left-turn capacity of the intersection and the length of reverse-variable lane. This phenomenon is obvious at the short-link grouped intersections[6].Therefor, this paper choice the length of short-link grouped intersections equal to two hundred meters[7].

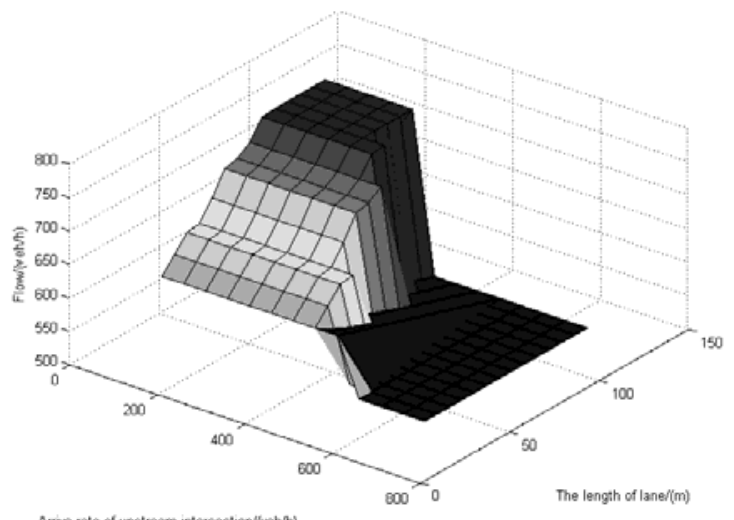

FIGURE VI RELATIONSHIP BETWEEN THE REVERSE TRAFFIC ARRIVE RATE OF UPSTREAM INTERSECTION AND LEFT-TURN PASTED-TRAFFIC FLOW

From Figure.6, we can note that the left-turn capacity keep a certain value with the increasing of arrive rate of upstream intersection, when the length of reverse-variable lane is not been changed, at the beginning. But the left-turn capacity descent steeply into a certain number $(d)$, when the arrive rate of upstream intersection reach a critical value(e). By contrasting with Figure.3, we found that the value of curve which is unset reverse-variable lane, is same with $d$, which mean that reverse-variable lane does not make any mean. By analyze, we find the reason is that the queuing vehicles is so long that make the entrance of reverse-variable lane blocked. Therefor, we can decide the length of reverse-variable lane on the base of $e$, in order to make the reverse-variable lane work well, in reality.

\section{CASE SimULATION}

Taking the intersection of the Great Wall Road and Phoenix Street in Yinchuan City as an example, this paper make a case simulation.

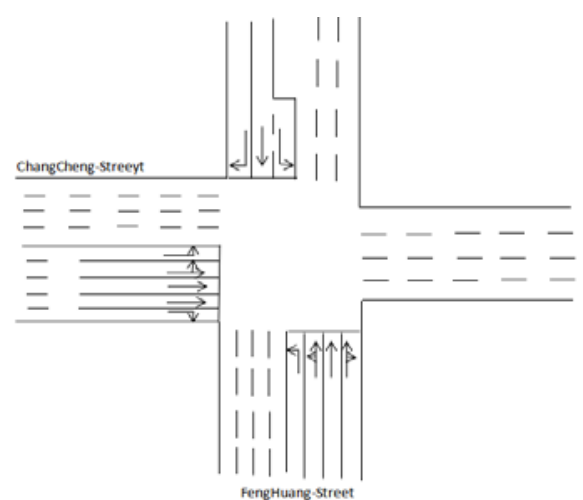

(a) 


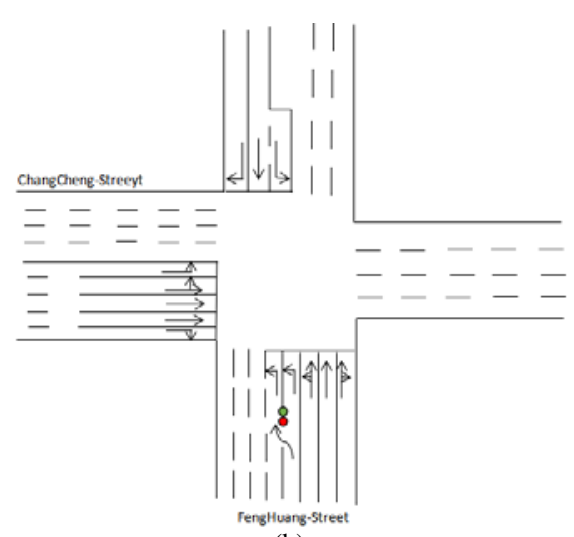

(b)

\section{FIGURE VII THE INTERSECTION OF} CHANGCHENG-STREET AND FENGHUANG-STREET

Figure.7 is the road channelization diagram of the intersection, Figure. 7(a) is the road channelization diagram when the reverse variable lane is not set, and Figure. $7(b)$ is the road channelization diagram after a reverse variable lane is set. Table. 1 shows the traffic statistics measured at the late peak of the intersection when there is no reverse variable lane. The timing scheme is as follows: Weststraight fifty five seconds, West -left twenty three seconds, South-North straight forty four seconds, South-North left thirty six seconds, cycle one hundred and fifty eight seconds. The main traffic problems are: there are three queues for the left turn vehicles in the south entrance, seventeen to twenty three vehicles in a single cycle left turn through, six to ten vehicles not cleared, thirty five queues and the phenomenon of queuing up to the upstream railway section, due to southward entry During the rush hour, the traffic volume of left turn is large, and there is a straight left lane, which also affects the traffic of the direct traffic in the south entrance.

TABLE I. THE VOLUME MEASURED OF CHANGCHENG-STREET AND FENGHUANG-STREET IN TOWARD EVENING RUSH HOUR

\begin{tabular}{|c|c|c|c|c|c|c|}
\hline \multirow{2}{*}{\multicolumn{3}{|c|}{$\begin{array}{l}\text { Time } \\
\text { Quantum Direction Flow-direction }\end{array}$}} & \multicolumn{3}{|c|}{ Type of car } & \multirow{3}{*}{$\begin{array}{l}\text { Subt } \\
\text { otal } \\
\text { equi } \\
\text { vale } \\
\text { nt }\end{array}$} \\
\hline & & & \multirow{2}{*}{$\begin{array}{l}\begin{array}{l}\text { Sed } \\
\text { an }\end{array} \\
316\end{array}$} & \multirow{2}{*}{$\begin{array}{l}\text { Medi } \\
\text { um } \\
\text { type } \\
\text { car } \\
16 \\
\end{array}$} & \multirow{2}{*}{$\begin{array}{l}\begin{array}{l}\text { Ove } \\
\text { rsize } \\
\text { vehi } \\
\text { cle }\end{array} \\
89 \\
\end{array}$} & \\
\hline \multirow{12}{*}{$\begin{array}{l}\text { Toward } \\
\text { evening } \\
18: 00-1 \\
9: 00\end{array}$} & & Left-turn & & & & \\
\hline & \multirow{3}{*}{$\begin{array}{l}\text { Wester } \\
\text { n } \\
\text { import }\end{array}$} & Go -Straight & 150 & 18 & 260 & 1785 \\
\hline & & Right-turn & 312 & 1 & 19 & 332 \\
\hline & & Turn-round & 51 & 0 & 0 & 51 \\
\hline & \multirow[b]{4}{*}{$\begin{array}{l}\text { Souther } \\
\mathrm{n} \\
\text { import }\end{array}$} & Left-turn & 412 & 11 & 71 & 494 \\
\hline & & Go -Straight & 803 & 23 & 89 & 915 \\
\hline & & Right-turn & 180 & 0 & 19 & 199 \\
\hline & & Turn-round & 27 & 1 & 0 & 28 \\
\hline & \multirow{4}{*}{$\begin{array}{l}\text { North } \\
\text { import }\end{array}$} & Left-turn & 239 & 2 & 51 & 292 \\
\hline & & Go -Straight & 765 & 9 & 86 & 860 \\
\hline & & Right-turn & 101 & 0 & 13 & 114 \\
\hline & & Turn-round & 19 & 1 & 0 & 20 \\
\hline
\end{tabular}

By using Vissim traffic simulation software to set up the ChangCheng-Street and FengHuang-Stree's intersection model with the substitute the measured traffic data from Table.1. The traffic scene without reverse variable lane is simulated, and the simulation results are consistent with the actual traffic volume. According to the method described above, simulating the traffic scene shown in Figure.7(b), the simulation results are as follows: when the timing scheme is not changed, there is an optimal reverse variable lane length of twenty five meters, which can make the south entrance pass through thirty five vehicles in a cycle. In one cycle, the queued vehicles are cleared completely, and the capacity of left turn increases 52.3\%. The south entrance of the intersection is actually set up with a reverse variable lane length of thirty five meters. The simulation method is introduced in 2.3 above, and the length of the reverse variable lane is thirty five meter. The result is as follows: the time of the left turn green light at the south entrance is compressed to thirty seconds, a cycle passes through thirty five vehicles, and the queue of vehicles can be completely empty in one cycle. The saved six seconds can be allocated to other phases, or compression cycle six seconds to achieve faster rotation.

\section{CONCLUSIONS}

This paper researched the influence that the arrive rate, green time, the length of reverse-variable lane and the arrive rate of upstream intersection in short-link grouped intersection make on capacity, by cellular automata model. By analyze the numerical simulation result, we drew the conclusion that there are a critical value of arrive rate to set reverse-variable lane, a most suitable length of reverse-variable lane, and a critical value of the arrive rate of upstream intersection, which may make the entrance of reverse-variable lane blocked. Therefore, the optimal reverse variable lane can be set according to the arrival rate of the reverse traffic flow at the upstream intersection. With the increase of the length of the left turn phase green light, the optimal reverse variable lane length will be reduced. Therefore, the reverse variable lane length must be reset when the intersection timing changes. When the length of the reverse variable lane is fixed, there exists a green light time which can make the traffic flow pass to the maximum, thus avoiding the waste of the timing caused by the excessive allocation of the green time. The research in this paper has certain guiding significance to the reality. Taking the intersection of ChangCheng-Street and FengHuang-Street in Yinchuan City as an example, it is verified that the simulation method proposed in this paper can effectively guide the reverse intersections. Setting of variable lane and optimization of timing scheme.

\section{ACKNOWLEDGMENT}

Thsnks the excelent education of my school, and this cherished chance of AMMSA 2018 CONFERENCE.

\section{REFERENCES}

[1] SUN Zheng-ming, YANG Xiao-guang. Methods for Spatial Layout Design of Urban Street at -Grade Intersections[J]. Urban Transport of Chain, 2006, 4(3): 47-52. 
[2] SHANG Zhen-hua. Setting Method of the Reverse Variable Lane in the Urban Intersections[D]. CHANG'UN UNIVERSITY, 2013.

[3] WANG Jing-yuan, WANG Wei. Calculation of storage lengths for left-turn lanes with permitted phases[J]. CHINA CIVIL ENGINEERING JOURNAL, 2011, 44(9): 114-123.

[4] YANG Xiaoguang, ZHAO Jin, MA Wangjin, et al. Reviews oncalculation method for signaled intersection capacity[J]. ChinaJournal of Highway and Transport, 2014, 27(5): 148-157.

[5] Hummer J E, Montgomery R E, Sinha K C. Guidelines for use of leading and lagging left-turn signal phasing [J]. Transportation Research Record, 1991, 1324(3): 11-20.

[6] LV Bin, NIU Hui-min. Signal timing optimization at isolated intersections under random condition[J]. Journal of Traffic and Transportation Engineering, 2010 ,10(6): 116-120.

[7] LIU Ying-dong, NIU Hui-min, WANG Jian-qiang. Cellular Automata Model Short-link Grouped Intersections[J]. Journal of Highway and Transportation Research and Development, 2015, 32(2): $140-146+153$ 